\title{
IMPLANTAÇÃO DA METODOLOGIA DIAGNOSIS RELATED GROUPS NA FUNDAÇÃO HOSPITALAR DO ESTADO DE MINAS GERAIS: UM ESTUDO DE CASO
}

\section{IMPLEMENTATION OF THE DIAGNOSIS RELATED GROUPS METHODOLOGY IN THE HOSPITAL FOUNDATION OF THE STATE OF MINAS GERAIS: A CASE STUDY}

\section{IMPLEMENTACIÓN DE LA METODOLOGÍA DE GRUPOS RELACIONADOS CON EL DIAGNÓSTICO EN LA FUNDACIÓN HOSPITALARIA DEL ESTADO DE MINAS GERAIS: UN ESTUDIO DE CASO}

\author{
Barbara Ribeiro Martins \\ Coordenadora do Projeto Diagnosis Related Groups (DRG) na Fundação Hospitalar do Estado de Minas \\ Gerais. Mestrado em Enfermagem pela UFMG. \\ MBA Acreditação em Saúde pela Faculdade de Ciências Médicas de Minas Gerais, FELUMAl. \\ Especialização em Qualidade e Segurança no Cuidado ao Paciente, pela Sociedade Beneficente de Senhoras \\ Hospital Sírio-Libanês. \\ Graduação em Enfermagem pela PUC-Minas e Graduação em Gestão de Serviços de Saúde pela UFMG. \\ Fundação Hospitalar do Estado de Minas Gerais - FHEMIG, Belo Horizonte, MG, Brasil \\ Marco Aurélio Fagundes Ângelo \\ Coordenador Médico do DRG da Fundação Hospitalar do Estado de Minas Gerais. \\ Mestrado em Administração pela Universidade Federal de Minas Gerais. \\ Graduação em Medicina pela Universidade Federal de Minas Gerais (I990) e MBA pela FGV / Ohio University, \\ atuando principalmente em gestão hospitalar e de organizações de saúde. Médico especialista em anestesiologia (MEC / \\ TSA-SBA) e especialista em medicina intensiva (AMIB). \\ Fundação Hospitalar do Estado de Minas Gerais, Belo Horizonte, MG, Brasil
}




\section{RESUMO}

O objetivo geral deste estudo é descrever a implantação da metodologia DRG na Fundação Hospitalar do Estado de Minas Gerais. Os objetivos específicos são: descrever como ocorreu o planejamento, definição e operacionalização dos macroprocessos e atividades do sistema DRG em IO hospitais da Fhemig, apresentar resultados iniciais da utilização da solução. Trata-se de um estudo de caso. O resultado foi: a implantação do DRG integra três macroprocessos que são a Definição da Política e Diretrizes Hospitalares; a Ferramenta de Gestão Hospitalar; e a Estruturação e Manutenção do Banco de Dados, com a instituição de um modelo de governança. Destacam-se os indicadores de Média de Permanência Geral com DRG e o Percentual de Altas Codificadas. Os resultados iniciais apresentam os indicadores de complexidade e desempenho hospitalar da Fhemig, além de compará-los com o SUS, Saúde Suplementar e Assistência Privada, evidenciando o vocacionamento para especialidades com tendência para case mix maiores na Fhemig. Conclui-se que, com o processo de implantação, foi possível realizar um diagnóstico do perfil assistencial e do desempenho.

PALAVRAS-CHAVE: Política de Saúde, Administração hospitalar, Grupos de Diagnósticos Relacionados.

\section{ABSTRACT}

The general goal of this study is to describe the implementation of the DRG methodology at the Hospital Foundation of the State of Minas Gerais. The specific goals are: to describe how the planning, definition and operationalization of the DRG system's macro processes and activities in IO Fhemig hospitals, and to present initial results of the use of the solution. This is a case study. The result was: the implementation of the DRG integrates three macro processes, which are Definition of Hospital Policy and Guidelines; Hospital Management Tool; and Database Structuring and Maintenance, with the institution of a governance model. The indicators of Overall Permanence Average with DRG and the Percentage of Coded Discharges stand out. The initial results present Fhemig's hospital complexity and performance indicators, in addition to comparing them with SUS, Supplementary Health and Private Assistance, evidencing Fhemig's vocation for specialties with a tendency towards larger case mixes. It is concluded that, with the implementation process, it was possible to carry out a diagnosis of the care profile.

KEYWORDS: Health Policy, Hospital Administration, Diagnosis Related Groups.

\section{RESUMEN}

El objetivo general de este estudio es describir la implementación de la metodología DRG en la Fundación Hospitalaria del Estado de Minas Gerais. Los objetivos específicos son: describir cómo se llevó a cabo la planificación, definición y ejecución de operaciones de los macroprocesos del sistema DRG en IO hospitales; y presentar los resultados iniciales del uso de la solución. Este es un estudio de caso. El resultado obtenido fue que la implementación del DRG integra tres macroprocesos: La definición de Política y Lineamientos Hospitalarios; La herramienta de Gestión Hospitalaria; y La estructuración y mantenimiento de base de datos con la institución de un modelo de gobernanza. Se destacan los indicadores de Promedio de Permanencia General y el Porcentaje de codificación. El diagnóstico del perfil asistencial presenta los indicadores de complejidad y desempeño hospitalario de la FHEMIG, además de compararlos con el Sistema Único de Salud, Salud Complementaria y Asistencia Privada, evidenciando la vocación por las especialidades con tendencia a mayores grupos Case Mix. Se concluye que con el proceso de implementación fue posible realizar un diagnóstico del perfil de atención.

PALABRAS CLAVE: Política de Salud, Administración Hospitalaria, Grupos de diagnóstico relacionados. 


\section{INTRODUÇÃO}

A implantação do Sistema DRG na Fundação Hospitalar do Estado de Minas Gerais se insere no contexto da política, planejamento e administração em saúde e, em especial, na atenção hospitalar.

Segundo Paim e Teixeira (2006), a política de saúde é a resposta social de uma organização frente às condições de saúde dos indivíduos, que envolve a produção, distribuição, gestão e regulação dos bens e serviços que de alguma forma possuem ligação à saúde humana e ao ambiente. No Sistema Único de Saúde (SUS), a política de saúde envolve um conjunto coordenado de ações, planejamento estratégico e tomada de decisão pelos gestores públicos das três esferas de governo - municipal, estadual e federal.

No âmbito do SUS, a Rede de Atenção à Saúde (RAS) foi instituída pela Portaria GM/MS n 4.279 , de 30 de dezembro de 2010, como estratégia para superar a fragmentação da atenção e da gestão nas Regiões de Saúde e aperfeiçoar o funcionamento político-institucional do sistema público, com vistas a assegurar ao usuário o conjunto de ações e serviços que necessita com efetividade e eficiência. Visando o aprimoramento do SUS e das RAS, instituiu a Portaria de Consolidação n ${ }^{\circ}$ 03, de 28 de setembro de 2017, e definiu as normas para consolidação das redes do Sistema Único de Saúde (BRASIL, 20I0; BRASIL, 20I7a).

Em relação à atenção hospitalar, o principal marco normativo é a Política Nacional de Atenção Hospitalar (PNHOSP), instituída pela Portaria MS/GM 3390/2013, seguido da Portaria de Consolidação $\mathrm{n}^{\circ} 2$, de 28 de julho de 2017 , que dispôs sobre a consolidação das políticas nacionais de saúde, e estabelece também as diretrizes para a organização do Componente Hospitalar (BRASIL, 20I3; BRASIL, 2017b).

A atenção hospitalar é uma temática importante na análise de políticas públicas de saúde. Seu fomento é histórico no Brasil, o que contribuiu de maneira fundamental para o modelo assistencial médico hospitalocêntrico. Assistência hospitalar é emblemática no campo da política, planejamento e gestão e constitui-se um objeto de constante preocupação por parte dos gestores, tendo em vista a complexidade e os desafios existentes na área (SANTOS; PINTO, 2017).

Carpanez e Malik (202I) apontam que a Política Nacional de Atenção Hospitalar iniciou a reorganização do sistema, mas discussões e ações ainda são necessárias e de forma veemente. $O$ reordenamento dessa rede implica em garantia do acesso, integralidade do cuidado, qualidade das ações e eficiência do sistema. As mesmas autoras problematizam as transformações necessárias frente à capacidade instalada existente, bem como quais são as perspectivas e modelos gerenciais propostos para atenção hospitalar no Brasil.

Os hospitais são instituições complexas, com densidade tecnológica específica, de caráter multiprofissional e interdisciplinar, responsável pela assistência aos usuários com condições agudas ou agudização de crônicas, que apresentem potencial de instabilização e de complicações de seu estado de saúde, exigindo-se assistência contínua em regime de internação (BRASIL, 2017b).

Os hospitais podem ser caracterizados, na perspectiva da RAS, por pontos de atenção secundários e terciários em que se ofertam determinados serviços especializados, gerados através de uma função de produção singular. Eles se diferenciam por suas respectivas densidades tecnológicas, sendo os pontos de atenção terciários mais densos tecnologicamente que os pontos de atenção secundários e, por essa razão, tendem a ser mais concentrados espacialmente (MENDES, 20I0).

Existe um consenso de que a melhoria na eficiência do parque hospitalar seja prioridade dos governos. Nos sistemas de saúde, os hospitais se apresentam como estruturas fundamentais, executando a maior parte das ações de prevenção secundária (diagnóstico precoce e tratamento imediato) e terciária (prevenção de incapacidades e reabilitação). Compreender os sistemas hospitalares pode fornecer subsídios para melhorar a eficiência, a eficácia e a efetividade nas ações de saúde, bem como aprimorar o planejamento nas ações dos sistemas de saúde (CARPANEZ; MALIK, 202I).

Ugá (2012) aponta que, para gerenciar o sistema de saúde a partir de redes integradas de serviços, faz-se necessário a utilização de instrumentos de regulação dos prestadores de serviços de saúde, tais como modelos de contratualização entre o gestor público e prestadores, bem como novos sistemas de 
remuneração aos prestadores de serviços que atrelem o pagamento por serviços prestados com a avaliação de qualidade dos serviços prestado.

Nesta vertente de discussão, a metodologia Diagnosis Related Groups (DRG) se apresenta com uma possibilidade de avaliação da qualidade do cuidado e da eficiência na utilização de recursos e serviços no ambiente hospitalar.

O DRG é um sistema de classificação de pacientes construído na perspectiva de instrumentalizar a gestão hospitalar. Essa classificação, desenvolvida na Universidade de Yale, nos Estados Unidos, no final da década de 1960 e ao longo da década de 1970, teve o objetivo de definir o produto hospitalar para fins de monitoramento da utilização de serviços, bem como de avaliação, remuneração e gerência de qualidade da atenção hospitalar (FETTER, I99I).

O desenvolvimento e utilização nos EUA suscitou interesse pelo sistema DRG no contexto internacional e pesquisas foram realizadas com o intuito de avaliar a formação de DRGs a partir de dados disponíveis e verificar os usos possíveis deste sistema para hospitais quanto no sistema de saúde como um todo. (NORONHA et al., I99I).

No âmbito da gestão estadual, destaca-se a Fundação Hospitalar do Estado de Minas Gerais (Fhemig) como uma das maiores gestoras de hospitais públicos do país com a prestação de serviços hospitalares de diversas especialidades. A Fhemig administra 2I unidades assistenciais, das quais I0 hospitais foram contemplados no Projeto DRG-Fhemig e passaram a compor base de dados do Sistema DRG Brasil.

O Projeto DRG-Fhemig foi proposto em um contexto institucional em que as atividades de monitoramento do desempenho hospitalar baseavam-se em metas de melhoria da série histórica dos indicadores de Média de Permanência e Taxa de Ocupação, geral e específica, restringindo o benchmark, quando ocorria, em indicadores de estrutura, produção, pessoal e custos.

Identificou-se então, a necessidade de aprimorar o monitoramento e avaliação, pelos parâmetros de complexidade dos pacientes atendidos e de desfechos assistenciais, de forma comparativa com as boas práticas assistenciais e com resultados obtidos por outros serviços hospitalares. Dessa maneira, a possibilidade de inserir a metodologia DRG na Fundação preencheria a lacuna supracitada para avaliação de desempenho ao ajustar indicadores pelo Índice de Complexidade dos Pacientes.

O objetivo geral deste estudo é descrever a implantação da metodologia DRG na Fundação Hospitalar do Estado de Minas Gerais. Os objetivos específicos são: descrever como ocorreu o planejamento, definição e operacionalização dos macroprocessos e atividades do sistema DRG em I0 hospitais da Fhemig; e apresentar resultados iniciais da utilização da solução.

A relevância da implantação do sistema DRG está relacionada à definição de políticas de investimento e gestão eficiente de recursos, além de garantir uma transparência dos dados que refletem a complexidade e qualidade dos serviços hospitalares ofertados à população. Ademais, a implantação do DRG na Fhemig e os resultados parciais já alcançados refletem uma mudança de paradigma no planejamento, monitoramento e avaliação dos serviços hospitalares. Por fim, a publicação dos resultados deste estudo justifica-se também pelo uso incipiente do DRG para formulação de políticas de saúde e para ferramenta de gestão hospitalar no SUS, e por ser um tema com escassez de publicação no cenário nacional.

\section{FUNDAMENTAÇÃO TEÓRICA}

\section{A Metodologia Diagnosis Related Groups (DRG) o Desempenho Hospitalar}

O propósito inicial que motivou a criação do Diagnosis Related Group ( $D R G$ ), na década de 1960, foi desenvolver um sistema de monitoração da qualidade do cuidado e da eficiência na utilização de recursos e serviços no ambiente hospitalar. 
Nos Estados Unidos da América, o Medicare foi estabelecido em I965, e o Congresso Americano utilizou o sistema de "reembolso retrospectivo baseado em custos", modelo no setor de seguros privados, para pagamento dos hospitais. Ao final dos anos fiscais, hospitais apresentavam seus relatórios de custos, estes eram reconciliados com os "custos permitidos" definidos politicamente e por contrato. No período de 1967 a 1983 esses custos aumentaram de US\$ 3 bilhões para US\$ 37 bilhões, anualmente (CENTERS FOR MEDICARE \& MEDICAID SERVICES, 2000).

Considerando o período de 1967 a 1985, o número de matriculados no Medicare cresceu de I9,5 milhões para 3I,I milhões (59,49 \%), enquanto os gastos do sistema saltaram de US\$ 4,7bilhões para US\$ 72,3 bilhões (I538,30\%), e o percentual de gastos diretos com saúde passaram de $9,2 \%$ em 1967, para 16,9\% em I 985 (CENTERS FOR MEDICARE \& MEDICAID SERVICES, 200I).

Foi diante da pressão sobre o sistema de saúde e da necessidade de melhor controle sobre os gastos públicos, que o grupo do Dr. Robert B. Fetter, do Departamento de Pesquisas Operacionais do Instituto para Estudos Sociais e Políticos do Centro para Estudos em Saúde da Yale University, foi demandado pelo Health Care Financing Administration, Department of Health, Education and Welfare. Com o apoio do Departamento de Saúde do Estado de Nova Jersey, o grupo de pesquisadores debruçouse sobre as questões relativas ao desempenho hospitalar, normalmente monitorada pelos indicadores do Tempo de Permanência, Taxas de Ocupação, Número de Admissões ou Altas, Taxa de Mortalidade e Custo por Paciente, procurando entender quanto das diferenças observadas entre os hospitais deviam-se à combinação dos casos atendidos e quanto correspondiam a diferentes práticas assistenciais, com o propósito de fornecer uma estrutura de tipos de caso que refletisse a complexidade relativa dos pacientes atendidos em uma instituição (FETTER, 1980).

O grupo de Fetter entendeu que os DRGs, conforme proposto à época, cumpriram os objetivos de fornecerem uma classificação de tipos de casos que retinham as propriedades pré-definidas (FETTER, 1980):

- As variáveis envolvidas para caracterização de um caso restringiam-se a condições clínicas dos pacientes e à assistência fornecida e eram de registro habitual nos sistemas de informação dos hospitais: diagnóstico primário, diagnósticos secundários, idades, procedimentos cirúrgicos;

- Foi gerado um número gerenciável de casos (383 DRGs, à época), sendo estes mutuamente exclusivos e exaustivos;

- Cada DRG possuía uma interpretabilidade clínica (era compreendido pela equipe assistencial) e possuía diagnósticos e procedimentos intimamente relacionados;

- Todos os pacientes alocados em um DRG compartilhavam padrões semelhantes de consumo de recursos, tempo de internação e desfechos.

O grupo de pesquisadores de Yale concluiu que o sistema de classificação do DRG certamente teria aplicações variadas no gerenciamento hospitalar, na formulação de políticas assistenciais relativas ao cuidado hospitalar, na regulação e auditoria de serviços hospitalares, na formulação de políticas remuneratórias para serviços hospitalares, na contabilidade, e todos caracterizados pela centralidade no paciente. A metodologia DRG, focada na complexidade e necessidade do paciente, asseguraria aos programas e serviços responsáveis por essas atividades que compartilhassem uma base conceitual comum (FETTER, 1980).

A evolução do DRG para um sistema que servisse de base ao Sistema de Pagamento Prospectivo por serviços de saúde foi consequência da sua capacidade de categorizar e valorar o "case mix" (população demandante sobre um serviço de saúde) do hospital ou sistema de saúde, além de relacioná-lo aos custos assistenciais, eficiência, efetividade e resultados obtidos. O Medicare adotou o DRG a partir de I983, passando a negociar e remunerar prestadores pelo Sistema de Pagamento Prospectivo, assegurando economias da ordem de US\$50 bilhões, desde sua adoção até I990 (FETTER, I99I). Portugal foi o primeiro país europeu a adotar o DRG, iniciando em 1988. Sua disseminação pela Europa, Austrália, Américas Central e do Sul vem ocorrendo ao longo dos últimos 30 anos, tornando o DRG o Sistema de Classificação de Pacientes mais importante e de maior utilização internacional (BUSSE, et. al; 20II). 
O processo de alocação de um determinado paciente é realizado pelo algoritmo de clusterização, através de um processo sequencial de tratamento dos atributos (variáveis) que caracterizam a condição do paciente, sua demanda por recursos assistenciais e sua interação com o provedor de cuidado (FETTER, I99I).

Essas variáveis são extraídas do prontuário do paciente e codificadas no sistema que possui o algoritmo de agrupamento, a partir de processos que variam de totalmente manuais a totalmente automatizados e digitais.

A primeira variável considerada é o Diagnóstico Principal, codificado através da Classificação Internacional de Doenças (CID), que na época da elaboração do DRG era a CID 9, estando em uso atualmente a CID I0. Em seguida, a partir do CID codificado como diagnóstico principal, o paciente era alocado em uma das 23 Categorias Diagnósticas Maiores (Major Diagnostic Categories - MDC) disponíveis, atualmente há 25 MDC, além de duas categorias especiais: -I Pré Grande Categoria; 0 - Sem MDC associado. O passo subsequente, é identificar se o paciente necessitou de algum procedimento cirúrgico, dividindo os grupamentos possíveis em clínicos (sem necessidade de cirurgias) ou cirúrgicos (paciente demandou algum procedimento cirúrgico). E, em sequência, considerando sequencialmente as variáveis, comorbidades e complicações, idade, condição de alta e necessidade de procedimentos não cirúrgicos (ventilação mecânica, diálise etc.), o paciente era finalmente alocado em um dos 383 DRGs disponíveis na época (FETTER, I99I).

A metodologia DRG prevê, não só a possibilidade de customização, como aconteceu com a criação de variantes específicas para sistemas de saúde, como na Alemanha, Austrália e outros países europeus, bem como sua evolução, conforme evoluam também os sistemas de codificação, com a necessidade de inclusão de novos códigos (ex: CID relacionados à COVID-I9 confirmada ou não confirmada laboratorialmente), ou pela incorporação de refinamentos que assegurem uma maior granularidade na caracterização do paciente (BUSSE, et. al; 20II), sendo a versão atual da Medicare Severity - DRG, a versão 38, Release I (CENTERS FOR MEDICARE \& MEDICAID SERVICES; 2019).

Assim, a versão brasileira do MS-DRG é a versão refinada, pois acrescenta subníveis de categorização de gravidade em múltiplos DRGs. Esta versão conta com $27 \mathrm{MDCs}$, três subcategorias quanto à presença ou não de comorbidades e complicações (sem comorbidades e complicações; com comorbidades e complicações; com comorbidades e complicações maiores), 754 DRGs e 1240 DRGs Refinados (subníveis de categorização de gravidade) para determinados DRG (Manual Operacional DRG Brasil - DRG Brasil Refinado).

No contexto nacional, Couttolenc e La Forgia (2008) em um extenso estudo sobre desempenho Hospitalar no Brasil patrocinado pelo Banco Mundial, deixam claro a grandiosidade do Sistema Hospitalar no Brasil e descrevem a centralidade dos hospitais no sistema público brasileiro, porque estes são, muitas vezes, a porta de entrada do cidadão no sistema de saúde, devido a falhas na Rede de Atenção Básica; por absorverem quase $70 \%$ dos gastos públicos com saúde; pelo seu peso político e midiático, por serem entendidos como centros de inovação tecnológica; e pela preocupação com seu desempenho (COUTTOLENC; LA FORGIA, 2008).

Ugá (20I2) relata que modelos remuneratórios variados têm sido testados, a metodologia DRG sendo um desses, com sucessos diferenciados na indução de eficiência e manutenção ou melhoria da qualidade dos serviços hospitalares na Comunidade Europeia. Ela reforça que modelos remuneratórios dependem de sistemas de classificação de pacientes para modelagem dos orçamentos hospitalares, e considera crítica a caracterização do case mix dos prestadores. No entanto, ela reforça que nenhum modelo remuneratório conseguiu assegurar a maior eficiência quantitativa aliada à maior eficiência qualitativa.

\section{METODOLOGIA}


Trata-se de um estudo de caso, descritivo. Neste estudo, o caso a ser analisado é a implantação da metodologia DRG na Fundação Hospitalar do Estado de Minas Gerais, uma das maiores gestoras de hospitais públicos do Brasil, criada em 1977, que administra 2I unidades assistenciais na Região Metropolitana de Belo Horizonte e no interior do Estado de Minas Gerais.

O universo da pesquisa é composto pela referida Fundação e os IO hospitais que integram sua rede: Hospital João XXIII, Hospital Infantil João Paulo II, Hospital Maria Amélia Lins, Hospital Júlia Kubitschek, Hospital Alberto Cavalcante, Maternidade Odete Valadares, Hospital Eduardo de Menezes, Hospital Regional Antônio Dias, Hospital Regional João Penido, Hospital Regional de Barbacena Dr. José Américo.

Estudos de caso são indicados para estudos organizacionais e gerenciais e permitem o uso de várias fontes de dados, desta maneira, ampliam as perspectivas de qualidade e riqueza de detalhes na descrição do caso (YIN, 20I0).

A coleta de dados foi realizada a partir de dados gerenciais relacionados ao processo de implantação. Foram utilizados documentos normativos, pareceres técnicos, matriz de indicadores, fluxogramas, procedimentos sistêmicos, procedimentos operacionais padrão, relatórios de avaliação de desempenho hospitalar e demais documentos pertinentes ao processo selecionado. Especificamente para apresentação dos resultados iniciais da utilização da solução, foram utilizados bancos de dados, relatórios e painéis do sistema DRG para traçar o referencial nacional e o perfil da complexidade e desempenho assistencial.

Os resultados serão apresentados no próximo tópico do artigo e esses foram organizados nas seguintes subdivisões: Implantação do Projeto DRG na Fhemig e Resultados iniciais da utilização da solução.

\section{ANÁLISE E DISCUSSÃO DOS RESULTADOS}

\section{Implantação do Projeto DRG na Fhemig}

As iniciativas de contratação do sistema DRG na Fhemig dataram no ano de 2018 com estudo de viabilidade do Projeto DRG na Fundação e discussão da relevância e benefícios desta implantação para Fundação, cuja missão é ofertar assistência de média e alta complexidade no SUS. Porém, ressalta-se que já se discutia, na Fhemig, a necessidade de aprimorar as estratégias de monitoramento do desempenho assistencial, em particular dos hospitais, permitindo integrar o desempenho quantitativo e qualitativo e atrelá-los à possibilidade de manter um benchmarking contínuo, em relação a instituições com públicoalvo e características similares, e a contratação de soluções que utilizavam a metodologia DRG integravam essas discussões.

Nesse contexto, a equipe técnica destinada a este projeto elaborou e submeteu o Projeto de Contrapartida intitulado Treinamento, Capacitação e Pesquisa na Fundação Hospitalar do Estado de Minas Gerais na utilização e obtenção de resultados através da metodologia e ferramenta DRG Brasil na Comissão de Avaliação de Ações de Ensino, Pesquisa e Inovação, uma vez que o modelo contratual e recurso utilizados para aquisição da licença do software foi, de contrapartida, do Convênio de Estágios ${ }^{1}$, e teve um custo total de $\mathrm{R} \$ 270.000,00$ (duzentos e setenta mil reais), para cinco anos de contrato, significando um custo anual de $\mathrm{R} \$ 54.000,00$ (cinquenta e quatro mil reais).

I Processo coordenado pela Diretoria de Gestão de Pessoas (DIGEPE) da Fhemig e as legislações que fundamentam são: Lei Federal n ${ }^{\circ}$ I I.788.2008 de 25/09/2008 que dispõe sobre o estágio de estudantes; Lei I2079, de I2/0I/I996 que dispõe sobre estágio para estudante em órgão e entidade da administração pública; Decreto 45036, de 04/02/2009 que dispõe sobre estágio para estudantes em órgãos e entidades da administração pública direta, autárquica e fundacional, no âmbito do Poder Executivo. 
Em 2019, essas iniciativas culminaram na aprovação por esta Comissão, e assinatura do Termo de Acordo de Execução de Serviços pela Presidência da Fhemig, Instituição de Ensino do convênio e Grupo IAG Saúde, empresa detentora da patente DRG Brasil².

Por se tratar de um projeto institucional e contemplar IO hospitais da Fundação, como ações iniciais foram realizadas o Simpósio de Lançamento do Projeto DRG na Fhemig, e treinamento introdutório para gestores e analistas de informação em saúde (codificadores) sobre o uso da ferramenta e as respectivas atribuições destes profissionais.

No ano de implantação do projeto, destacam-se como fatores dificultadores: desconhecimento por parte dos gestores e profissionais de saúde sobre a metodologia e uso do DRG para classificação de pacientes; avaliação de complexidade e desfecho dos casos agudos; alocação de profissionais para atividade de codificação; adesão postergada de alguns hospitais ao projeto; e ausência de integração do Sistema de Gestão Hospitalar com o Software DRG.

Como fator estruturante do projeto, é importante mencionar a criação da Assessoria Técnica DRG com atribuições de planejamento, coordenação e de diretrizes do uso do DRG na Fhemig; de padronização e fomento de boas práticas de codificação; de treinamento e qualificação; de auditoria da codificação; de análise de dados e avaliação de resultados; e de implementação das boas práticas da Lei Geral de Proteção de Dados. Assessoria Técnica DRG integra a Diretoria de Contratualização e Gestão da Informação (DCGI) da Fundação.

Para fins de planejamento, acompanhamento e operacionalização das ações, a assessoria técnica DRG sistematizou e organizou as principais atividades no âmbito da Fundação em três macroprocessos: Definição da Política e Diretrizes Hospitalares; Ferramenta de Gestão Hospitalar; e Estruturação e Manutenção do Banco de Dados DRG. A seguir, a descrição de cada macroprocesso com as principais atividades previstas e resultados obtidos, bem como o Modelo de Governança pactuado. A estruturação dos macroprocessos foi aprovada no âmbito da diretoria e presidência.

Figura I: Estruturação e Interação entre os Macroprocessos e o Modelo de Governança

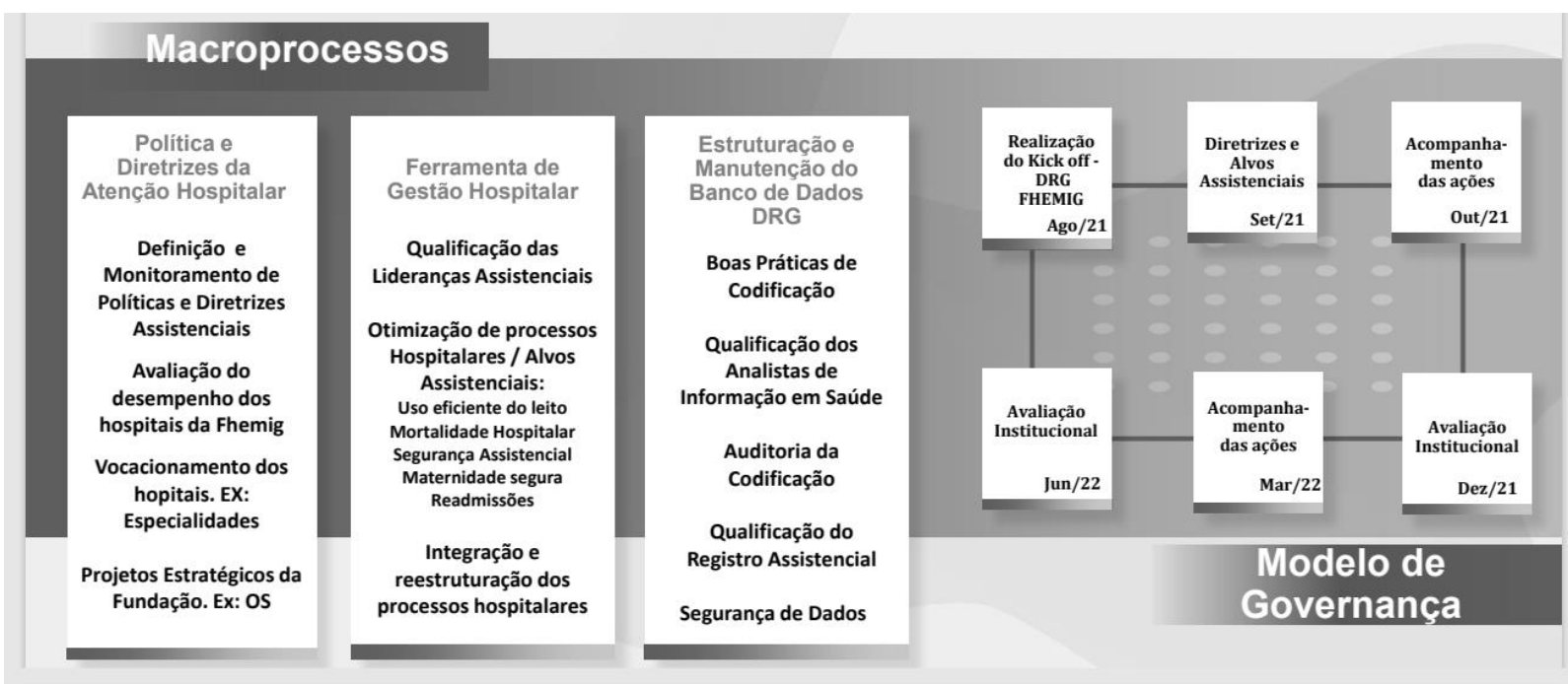

Fonte: Planejamento do Projeto DRG validado com a DCGI/Fhemig (compilado pelos autores).

A estruturação em macroprocessos é importante por se tratar de um projeto estratégico, abrangente e com processos em diferentes níveis gerenciais e operacionais da Fhemig e com resultados específicos, mas que, ao mesmo tempo, devem ser conduzidos de forma integrada e com visão sistêmica.

\footnotetext{
2 A metodologia DRG utilizada nos EUA foi adaptada ao Brasil no início dos anos 2000, por um grupo de pesquisadores vinculados à pós-graduação da Fundação Educacional Lucas Machado, a partir dos algoritmos para a classificação do Medicare Severity DRG (MS-DRG). O software DRG Brasil está em uso no país desde 201 I.
} 


\section{Política e Diretrizes da Atenção Hospitalar}

A utilização do DRG como ferramenta gerencial permite à Alta Direção da Fhemig a análise da base de Dados do Sistema DRG Brasil e se posicionar em relação a diferentes referências que compõem essa base (Benchmarking externo). Esse posicionamento possibilita uma análise conjunta ou individualizada de todos os hospitais da Fundação, e subsidia a elaboração de políticas assistenciais, remuneratórias, organizativas, além de identificar potenciais e limitações da assistência na Fhemig, para melhor negociação com os diversos atores gestores do Sistema Único de Saúde, nas esferas municipais, estaduais e federais.

A utilização dos DRGs contribui para que a avaliação do desempenho hospitalar ganhe nova dimensão. Os hospitais podem conhecer melhor os tipos de pacientes que atendem, os custos incorridos no tratamento deles, oferecendo novos subsídios para o planejamento dos seus serviços. O sistema ainda possibilita comparações entre hospitais, regiões e países, em que se pode observar variações na utilização de serviços, no processo de tratamento e nos resultados (NORONHA et al., I99I).

Os principais resultados desse macroprocesso, até outubro de 202I, foram: definição como projeto estratégico com acompanhamento das entregas pela presidência; inclusão de dois indicadores em dois ciclos de planejamento no Pacto de Gestão, que é o principal instrumento de operacionalização do Planejamento Estratégico adotado no Governo de Minas Gerais; e instituição de um modelo de Governança do Projeto DRG, com pactuação entre a presidência e os diretores hospitalares da Fhemig.

No modelo de Governança do Projeto DRG estão previstos grupos de trabalho no âmbito central e hospitalar, com a realização de encontros específicos para discussões, encaminhamentos e avaliação de resultados do desempenho hospitalar apontados pela metodologia DRG. Os encontros acontecem em Colegiados de Diretrizes e Alvos Assistenciais, Acompanhamento das Ações e de Avaliação Institucional.

Na perspectiva da RAS, o exercício da governança implica no enfrentamento de questões políticas e estruturais do processo de regionalização, como as capacidades internas de gestão, a sustentabilidade financeira, a regulação da atenção e o estabelecimento de padrões de qualidade para a provisão de serviços, bem como os padrões de gestão e desempenho das unidades de saúde, entre outros. No processo de governança são utilizados instrumentos como: roteiros de diagnóstico, planejamento e programações, sistemas de informação e identificação dos usuários, normas e regras de utilização de serviços, processos conjuntos de aquisição de insumos, complexos reguladores, sistemas de certificação/acreditação, monitoramento e avaliação, comissões/câmaras técnicas temáticas. (BRASIL, 20I7)

Vale ressaltar que o modelo de governança proposto no referido projeto é transversal aos macroprocessos Política e Diretrizes da Atenção Hospitalar e Ferramenta de Gestão Hospitalar. A seguir será descrito o escopo de atuação dos hospitais.

\section{Ferramenta de Gestão Hospitalar}

A utilização do DRG como metodologia pela Alta Direção e coordenações dos hospitais permite a avaliação dos processos assistenciais e gerenciais, através de um referencial adequado, quanto ao seu desempenho, qualidade do cuidado fornecido, potenciais e limitações que definem estratégias mais precisas e assertivas de intervenções.

Os gestores hospitalares, ao serem qualificados para a utilização do Sistema DRG, estão aptos a parametrizar e gerar, de forma customizada, os relatórios por este oferecidos, passando a dispor de um diagnóstico acurado do perfil de seus pacientes, desempenho e qualidade assistenciais. Dispondo desse diagnóstico, preciso e amplo, é possível maior assertividade no planejamento e tomadas de decisões, direcionando de forma mais específica as ações corretivas e de melhorias.

As análises oferecidas pelo Sistema DRG estão organizadas por alvos assistenciais: Uso Eficiente do Leito; Mortalidade Hospitalar; Segurança Assistencial; Maternidade Segura; Readmissões; Idoso Frágil; e Internações por Condições Sensíveis à Atenção Primária (ICSAP). Esses relatórios asseguram 
uma avaliação sistêmica do hospital, que pode ser detalhada para cada um destes alvos garantindo as ferramentas essenciais ao desenvolvimento do processo de governança clínica.

A metodologia DRG e o uso da solução Sistema DRG Brasil estão implantados na Fhemig, a utilização dos recursos ofertados por esta solução e o conhecimento da metodologia seguem amadurecendo. Como próximas fases do Projeto DRG Fhemig estão capilarizar o uso da metodologia DRG nos hospitais, e desenvolver o processo de Governança Clínica integrado à gestão de cuidados e de casos na Fhemig.

O DRG permite auxiliar a gestão econômica e clínica dos casos, por meio de indicadores de qualidade e segurança do paciente; adoção de protocolos e diretrizes clínicas baseados em evidência científica; acompanhamento de dados demográficos, epidemiológicos e de complexidade assistencial (ANS, 2019).

Em relação à qualificação dos gestores, foram realizados dois encontros com a alta direção de cada hospital para demonstrar e discutir como parametrizar e gerar esses relatórios. Como produto desta qualificação, está a elaboração do Diagnóstico do Desempenho Hospitalar a partir dos principais relatórios/alvos assistenciais do DRG. O próprio hospital será responsável pela elaboração deste e poderá propor intervenções hospitalares (ações corretivas ou de melhorias) e executá-las, bem como identificar e monitorar o impacto das ações e aprendizados obtidos.

Como ação gerencial de definição no âmbito das políticas, mas de impacto direto nos hospitais, foi estabelecido a inclusão do indicador de Média de Permanência Hospitalar pelo sistema DRG no Pacto de Gestão 2020-202I.

O indicador Média de Permanência Hospitalar vincula-se a um cronograma de metas:

- Bimestres I e 2: Efetuar o diagnóstico do desempenho assistencial em relação ao Percentil 50 da MP da base nacional do Sistema DRG Brasil, para Hospitais SUS de mesmo Case Mix, e elaboração do plano de ação para o progressivo alcance de desempenho (Média de Permanência) igual ao Percentil 50 de desempenho obtido pelo conjunto de referência.

- Os bimestres subsequentes vinculavam-se ao progressivo alcance do desempenho da MP do referencial: $25 \%, 50 \%, 75 \%$ e $100 \%$.

No período de vigência 202I/2022 do pacto de gestão, o Uso Eficiente do Leito permanece como alvo assistencial prioritário e obrigatório e é monitorado através do Indicador Média de Permanência Hospitalar, com novo cronograma de metas:

Para o bimestre I a meta é 60\%, e sequencialmente: 70\%, 80\%, 90\%, 95\% e 100\% da MP do Percentil 50 do desempenho (Média de permanência) da base nacional do Sistema DRG Brasil de Hospitais SUS de mesmo Case Mix. No ciclo de planejamento referido, também está previsto o indicador Média de Permanência por grupos de DRGs ou MDCs, visando uma maior assertividade das intervenções nos processos hospitalares. Esse indicador contribui para o direcionamento de intervenções nesses agrupamentos a partir da avaliação da prevalência, segundo o mesmo referencial do indicador de MP hospitalar.

As recomendações do uso eficiente do leito como alvo assistencial prioritário e o Indicador de Média de Permanência justificam-se pela indicação de única métrica de avaliação do desempenho hospitalar, mas que inclua atributos de eficiência e eficácia. Os hospitais da Fhemig, ao garantirem o adequado tempo de permanência de cada usuário, promovem um aumento de eficiência e redução de significativo potencial de complicações e custos, bem como a ampliação de acesso da população ao se otimizar a disponibilidade de leitos para o Sistema Único de Saúde.

\section{Estruturação e Manutenção do Banco de Dados DRG}

A consolidação do processo de codificação nos I0 hospitais possibilitou a estruturação de uma base de dados fidedigna e representativa para realização do diagnóstico do perfil assistencial da Fhemig.

A atividade da codificação é considerada crítica para a cadeia da informação DRG e, por isso, foi estabelecida a inclusão do indicador de Percentual da Codificação no Pacto de Gestão 2020-202I. No 
primeiro ano de vigência deste indicador, a meta inicial foi de 70\% e com o progressivo alcance de I00\% em relação ao volume de altas, nos bimestres apurados. Como resultado, no período de 01/07/2020 à 3I /07/2020, obteve-se um percentual médio de codificação de $85 \%$ nos total de altas dos I0 hospitais.

No Pacto de Gestão 2021/2022, esse indicador tem como meta garantir um Percentual da Codificação superior a $90 \%$ das altas hospitalares.

A atividade de codificação é feita por meio dos analistas de informação em saúde (codificadores), que são profissionais de nível superior na área da saúde, capacitados na interpretação, análise e codificação de prontuários na metodologia DRG. Os analistas captam todos os dados de saúde do paciente disponíveis nos prontuários, garantindo não só a consistência do diagnóstico principal, bem como as comorbidades relacionadas à saúde, evolução da doença, os procedimentos realizados, a assistência ofertada, iatrogenias e causas externas que podem impactar o desfecho da internação hospitalar de cada paciente.

No processo de implantação do Projeto DRG na Fhemig, foi necessário primeiramente calcular o dimensionamento dos analistas a partir da série histórica do volume de altas. Além disso, foi estabelecida a relação de codificação de 2,5 prontuários/altas por hora de trabalho do analista. Atualmente na Fundação totalizam 35 codificadores, médicos, enfermeiros e fisioterapeutas; com carga horária distinta entre as categorias profissionais e servidores.

Para a inserção destes profissionais na atividade de codificação foi realizado treinamento introdutório no formato de educação à distância (EAD) ofertado pela empresa parceira, acompanhado de treinamento prático pela assessoria técnica da Fhemig. No treinamento prático era demonstrado e discutido todos os campos para lançamento de dados do paciente e da assistência no sistema DRG. Além disso, era demonstrado no Sistema Integrado de Gestão Hospitalar da Fundação como navegar e localizar os principais campos e informações que mensuram a complexidade dos casos e assistência realizada, tais como: evoluções, evoluções específicas de terapia intensiva e bloco cirúrgico, registro de alta, resultados de exames, entre outros.

Além dos treinamentos introdutórios, foram disponibilizadas 20 vagas para o Curso de Aperfeiçoamento em codificação hospitalar e análise de informações em saúde na metodologia dos grupos de diagnósticos relacionados. Vale ressaltar que o curso é reconhecido pela Sociedade Brasileira de Analistas de Informação em Saúde - SBAIS e a distribuição de vagas seguiu os seguintes critérios: primeiramente a indicação dos analistas da assessoria técnica DRG, e as demais vagas foram distribuídas em todos os hospitais considerando o volume de altas.

Para fomentar as boas práticas de codificação, foi estabelecido também um Fluxo de Assessoria de Codificação. Esse fluxo tem como principal objetivo auxiliar os codificadores dos hospitais sobre dúvidas assistenciais do processo de codificação. Está previsto pelo fluxo a consulta ao banco de dúvidas de codificação, por meio de um link compartilhado. O banco integra I57 dúvidas categorizadas por temáticas, além de proposta de interpretação. Este é um processo contínuo e dinâmico, pois dúvidas e respostas são acrescentadas a partir da demanda dos codificadores dos hospitais. Caso a dúvida não seja sanada pela consulta ao banco, a orientação é o envio desta para e-mail específico da Assessoria Técnica DRG.

A estruturação do fluxo de assessoria foi necessária, pois a implantação do projeto em IO hospitais abarcou um número expressivo de profissionais em estágio inicial da curva de aprendizado da codificação do DRG, somado a diferentes especialidades e perfis de pacientes atendidos na Fundação.

Como fomento às boas práticas de codificação, também foram elaborados documentos de Procedimento Sistêmico para Cadastro e Codificação do DRG, com a finalidade de orientar, normatizar e padronizar essa atividade na Fhemig.

Após o processo de estruturação das atividades relacionadas à codificação, em janeiro de 202I, teve início a atividade de auditoria de codificação. Esta foi implementada na assessoria técnica DRG a partir de uma avaliação gerencial do percentual de inconsistências das altas codificadas. É feito a verificação da Validação da Base de Registro do Paciente e da Análise Complementar, que tem como 
finalidade promover a melhoria contínua dos processos de codificação, bem como o aprimoramento das competências dos Analistas de Informações em Saúde dos hospitais.

A literatura aponta que, para a implementação do DRG, como variáveis de infraestrutura e processo de trabalho destacam-se a padronização e auditoria. A classificação de casos é uma tarefa complexa e pode ter atribuição de DRG aos pacientes internados que não correspondem exatamente ao seu perfil de complexidade, como os grupos de mais alta pontuação, no fenômeno denominado de DRG creep (ANS, 2019).

Por fim, é importante ressaltar que a Estruturação e Manutenção do Banco de Dados DRG constitui o alicerce para a acurácia do diagnóstico assistencial e de desempenho da Fhemig, que será descrito no tópico a seguir.

\section{Resultados iniciais da utilização da solução}

A utilização do DRG permite transformar dados assistenciais em informação gerencial de forma estruturada e homogênea. O monitoramento contínuo do desempenho hospitalar, a partir do DRG, fomenta a melhoria na gestão de leitos e na qualidade da assistência hospitalar, por garantir um referencial de comparabilidade dos resultados com unidades que sejam reconhecidas como modelos. A seguir será apresentado o Diagnóstico Assistencial por Complexidade e o Desempenho dos Hospitais da Fhemig, como resultados iniciais da utilização da solução.

Vale ressaltar que, as 60862 altas representam a assistência hospitalar realizada pelos Complexos de Urgência, de Especialidades e de Referência, configurando assim, contribuição parcial da Fhemig para o SUS de Minas Gerais. As unidades assistenciais do Complexo de Saúde Mental e Complexo de Reabilitação e Cuidados Integrados, e a assistência ambulatorial especializada não utilizam o sistema DRG, pois a metodologia se aplica prioritariamente a casos agudos ou agudização de condições crônicas, e ainda não foi validado para a assistência psiquiátrica ou ambulatorial.

Do total de 60862 internações ocorridas nos IO hospitais de OI de julho de 2020 a 3 I de julho de 202I, 85\% ( $\mathrm{n}=5 \mathrm{I} 733)$ foram codificadas.

A Tabela I apresenta os resultados do case mix, índice gerado pelo algoritmo do Sistema DRG Brasil que resume a complexidade dos pacientes atendidos, e a média de permanência geral dos hospitais Fhemig. A variável de Ineficiência corresponde à razão entre as Médias de Permanência dos hospitais Fhemig (MP realizada) e a Média de Permanência prevista (MP prevista), considerando-se os DRGs que compõem o case mix da instituição, a partir do Percentil 50 da base de dados do DRG Brasil. Espera-se que a ineficiência operacional do custo fixo dos hospitais seja igual ou inferior a $100 \%$.

Tabela I: Análise dos hospitais Fhemig: - Case Mix, Ineficiência do custo fixo, 0I/jul/2020 a $3 \mathrm{I} / \mathrm{jul} / 202 \mathrm{I}$

\begin{tabular}{|c|c|c|c|c|c|c|}
\hline $\begin{array}{c}\text { Código do } \\
\text { Hospital }\end{array}$ & $\begin{array}{c}\text { Altas } \\
\text { codificadas }\end{array}$ & $\%$ das altas & $\begin{array}{c}\text { Média de Permanência } \\
\text { Real }\end{array}$ & $\begin{array}{c}\text { Média de Permanência } \\
\text { Prevista }\end{array}$ & Case Mix & Ineficiência \\
\hline $\mathrm{I} 022$ & 3360 & $6,49 \%$ & $7,7 \mathrm{I}$ & 4,38 & $\mathrm{I}, 4 \mathrm{I} 37$ & $\mathrm{I} 76,24 \%$ \\
\hline $\mathrm{I} 024$ & $265 \mathrm{I}$ & $5, \mathrm{I} \% \%$ & $\mathrm{I} 0,0 \mathrm{I}$ & $\mathrm{II}, 07$ & $2,734 \mathrm{I}$ & $90,4 \mathrm{I} \%$ \\
\hline $\mathrm{I} 025$ & 2450 & $4,74 \%$ & $\mathrm{II}, 73$ & 5,07 & $\mathrm{I}, 605 \mathrm{I}$ & $23 \mathrm{I}, 42 \%$ \\
\hline $\mathrm{I} 027$ & 3382 & $6,54 \%$ & 6,33 & 4,88 & $\mathrm{I}, 22 \mathrm{I} 4$ & $\mathrm{I} 29,76 \%$ \\
\hline $\mathrm{I} 028$ & $87 \mathrm{I} 8$ & $\mathrm{I} 6,85 \%$ & 7,89 & 5,05 & $\mathrm{I}, 3583$ & $\mathrm{I} 56, \mathrm{I} \% \%$ \\
\hline $\mathrm{I} 029$ & 8769 & $\mathrm{I} 6,95 \%$ & $7, \mathrm{I} \%$ & 4,40 & $\mathrm{I}, 7332$ & $\mathrm{I} 63,07 \%$ \\
\hline $\mathrm{I} 030$ & 2747 & $5,3 \mathrm{I} \%$ & 5,48 & $\mathrm{I}, 84$ & $\mathrm{I}, 3507$ & $298,55 \%$ \\
\hline $\mathrm{I} 03 \mathrm{I}$ & 7259 & $\mathrm{I} 4,03 \%$ & 5,99 & 4,28 & $\mathrm{I}, 5746$ & $\mathrm{I} 40,08 \%$ \\
\hline $\mathrm{I} 032$ & 4744 & $9, \mathrm{I} \%$ & 7,90 & 4,89 & $\mathrm{I}, 4 \mathrm{I} 25$ & $\mathrm{I} 6 \mathrm{I}, 55 \%$ \\
\hline $\mathrm{I} 035$ & 7653 & $\mathrm{I} 4,79 \%$ & 3,69 & 2,55 & $\mathrm{I}, 06 \mathrm{I} 7$ & $\mathrm{I} 44,55 \%$ \\
\hline Total & $5 \mathrm{I} 733$ & $\mathrm{I} 00,00 \%$ & 6,93 & 4,53 & $\mathrm{I}, 4897$ & $\mathrm{I} 52,96 \%$ \\
\hline
\end{tabular}

Fonte: Sistema DRG Brasil, compilado pelos autores (outubro/202I). 
É relevante salientar que o período do recorte temporal apresenta a "contaminação" pela pandemia da COVID-I9 e seus efeitos sobre o Case Mix assistido, mas as análises não são invalidadas, posto que o referencial de comparação também não foi poupado dos efeitos da COVID-I9.

Como as análises baseadas na metodologia DRG observam a centralidade no paciente, no processo de alocação dos casos em grupamentos de DRG, é possível conduzir análises específicas sobre a assistência hospitalar na Fhemig, considerando exclusivamente os pacientes com diagnóstico de infecção pelo vírus SARS Cov 2 de sua base e do referencial, ou excluir estes pacientes mantendo-se todos os demais. No entanto, considerando-se que a assistência aos pacientes de COVID-I9 requer, não só especializações para atendimento direto as suas vítimas, como também acarreta efeitos múltiplos sobre toda a ecologia do sistema de saúde, entende-se ser relevante manter estes pacientes, tanto na base apresentada como nos referenciais nacionais.

Alguns valores observados para as variáveis de Média de Permanência (realizada e prevista), Case Mix, e Ineficiência, no conjunto dos hospitais da FHEMIG, demonstram uma variância acentuada, porém, a análise cuidadosa da variação do Case Mix entre estas unidades já nos permite supor diferentes perfis assistenciais. Tal suposição, para algumas dessas observações é correta, representada por o extremo inferior de case mix da tabela ser um Hospital Maternidade exclusivo, e o extremo superior um hospital totalmente direcionado a atenção exclusiva a pacientes com COVID-19, no contexto da pandemia, no período de recorte estudado.

As análises de desempenho baseadas na metodologia DRG, devem atrelar a comparação a instituições de Case Mix semelhante, quando análises mais refinadas permitem compreender o quanto das diferenças observadas nas variáveis de análises, de hospital para hospital ou de hospital para um conjunto de hospitais, podem ser atribuída proporcionalmente à diferença das práticas assistenciais; o quanto é proporcional à diferença atribuível ao grupo de casos e o quanto pode ser atribuído à interação entre as diferenças das práticas assistenciais e ao grupo de casos.

$\mathrm{Na}$ Tabela 2 constam as mesmas variáveis apresentadas na Tabela I, entretanto estratificadas, conforme os cenários: Sistema Único de Saúde, Saúde Suplementar e Saúde Privada. Com a estratificação é possível comparar os resultados de desempenho observados pelas unidades Fhemig e pelo conjunto dessas unidades, ensejando futuras comparações com Sistemas de Assistência à Saúde do tipo holding, que agrega e gerencia múltiplas unidades hospitalares em sua rede própria.

No geral, em comparação com os outros cenários, a Fhemig apresenta maiores MP e menor case mix, todavia, obtém melhor desempenho (menor ineficiência) se comparada com o SUS, SS e assistência privada, no geral. Ademais, a análise comparativa entre todos os cenários e o hospital I024 da Fhemig apresentou, concomitantemente, melhores desempenhos e maiores complexidades assistenciais, ou seja, menores médias de permanências para tratar pacientes com maiores complexidades clínicas. O hospital I029 apresenta um case mix maior e ineficiência menor, se comparado aos de todos os outros cenários.

Tabela 2: Desempenho comparativo dos hospitais FHEMIG ao referencial nacional de mesmo Case Mix na assistência ao SUS, Saúde Suplementar e Saúde Privada, 0I/jul/2020 a 3I/jul/202I

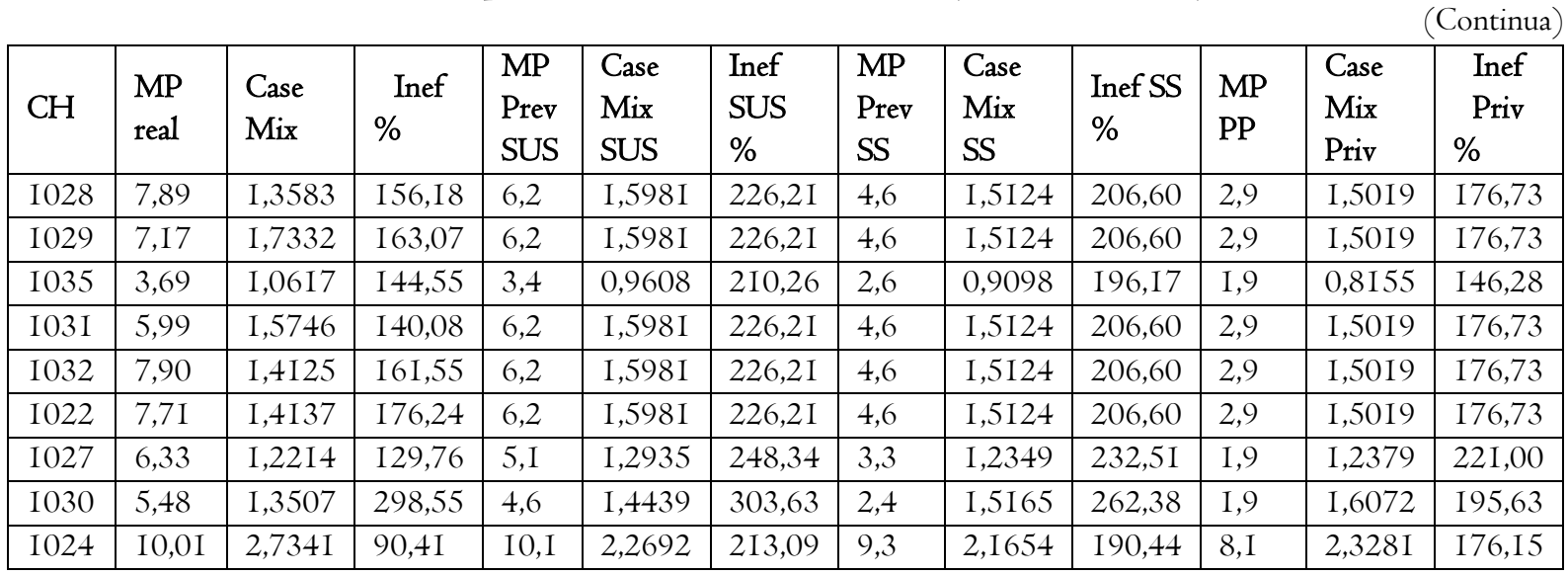




\begin{tabular}{|c|c|c|c|c|c|c|c|c|c|c|c|c|}
\hline \multicolumn{13}{|c|}{ (Conclusão) } \\
\hline I025 & II,73 & $\mathrm{I}, 605 \mathrm{I}$ & $23 \mathrm{I}, 42$ & 6,2 & I,598I & $226,2 \mathrm{I}$ & 4,6 & I,5I 24 & 206,60 & 2,9 & I,5019 & I76,73 \\
\hline Total & 6,93 & $\mathrm{I}, 4897$ & 152,96 & 5,0 & $\mathrm{I}, 5368$ & $2 \mathrm{I} 3, \mathrm{I3}$ & 4,6 & $\mathrm{I}, 5 \mathrm{I} 22$ & 206,10 & 2,9 & I,5019 & 176,73 \\
\hline
\end{tabular}

Fonte: Sistema DRG Brasil, compilado pelos autores (outubro/202I).

Legenda: $\mathrm{CH}$ : código do Hospital; MP real: Média de Permanência realizada; Inef\%: Ineficiência em percentual, MP Prev SUS: Média de Permanência prevista do SUS; Inef SUS \%: Ineficiência do SUS em percentual; MP Prev SS: Média de Permanência prevista da Saúde Suplementar; Case Mix SS: Case mix Saúde Suplementar; Inef SS \%: Ineficiência da Saúde Suplementar em percentual; MP PP: Média de Permanência prevista da Saúde Privada; Case Mix Priv: Case mix Saúde da Saúde Privada; Inef Priv\%: Ineficiência da Saúde Privada em percentual.

As variáveis de comparação (média de permanência prevista, case mix observado e ineficiência operacional sobre o custo fixo geral do conjunto das unidades) refletem os recortes, no mesmo período de análise das unidades da Fundação, da base nacional do DRG Brasil; porém, segmentadas por tipo de assistência, exclusiva ao SUS, da Saúde Suplementar ou do perfil exclusivamente privado.

Como são escassas publicações sobre a aplicação da metodologia DRG no monitoramento do desempenho hospitalar no SUS, as possíveis discussões à luz de publicações sobre o desempenho hospitalar no SUS ficam limitadas, em particular pelo uso de distintas ferramentas de análise.

Countollec e La Forgia (2008) afirmam que o desempenho nos hospitais públicos da administração direta frequentemente são os de pior desempenho. Porém, sem utilizar qualquer ferramenta de análise que considerasse a complexidade dos casos atendidos naquelas unidades, pois as ferramentas de análise se baseavam no uso do Data Envelopment Analisys, e nas variáveis usuais de desempenho, produção e custos, disponíveis à época.

Conforme Fetter (1980,199I) já apontava, mesmo utilizando rigorosamente as medidas de desempenho hospitalar, se não considerarem o conjunto das caraterísticas relevantes de complexidade e demanda por recursos assistenciais dos pacientes atendidos no serviço analisado, os resultados ficam desprovidos de contexto.

Com o uso da metodologia DRG e a partir dos resultados iniciais, verifica-se que para os hospitais Fhemig, públicos da administração direta, e os hospitais de atendimento exclusivamente SUS (não foram feitas parametrizações para o tipo de administração destes hospitais), existe uma significativa tendência para case mix maiores, refletindo uma maior complexidade da clientela assistida, em relação a hospitais que se situam dentro da mesma faixa de case mix, tanto da Saúde Suplementar e ainda mais, em relação à assistência privada.

A compreensão e análise dos fatores que podem contribuir para essa tendência fogem do escopo deste estudo de caso, mas certamente são influenciadas por muitos fatores que impactam a qualidade de vida dessa população, normalmente maiores vulnerabilidades e com restrições de acesso.

Comprovada essa tendência de concentração de um case mix mais complexo em favor dos hospitais exclusivamente SUS, e no caso da Fhemig, exclusivos SUS e da administração pública direta, é necessário estudar detalhadamente qual o percentual atribuível à diferença entre case mix, qual percentual é atribuível às diferenças dos padrões e processos assistenciais e quanto ainda poderia ser atribuível à interação entre esses dois componentes, como propõe Fetter (1980).

A Tabela 3 apoia a ideia do processo de clusterização sequencial da metodologia DRG. A estratégia para a composição desses dados é, após todo o processo de grupamento ter sido concluído, analisar os agrupamentos anteriores, porém com a composição do peso médio do DRGs para as categorias anteriores à alocação do DRG. As análises a partir das Categorias Diagnósticas Maiores (MDC - Major Diagnostic Categories) também são importantes, pois permitem um olhar sobre conjuntos de serviços hospitalares.

A Tabela 3 apresenta a descrição das Categorias Diagnósticas Maiores (MDC - Major Diagnostic Categories), para os casos clínicos e cirúrgicos, separadamente, dos 10 hospitais. Observa-se que a ineficiência dos casos cirúrgicos (I99,36\%) é superior se comparada aos casos clínicos (I44,30\%). Do total de casos clínicos $(\mathrm{n}=32048)$, destacam-se os seguintes MDCs, respectivamente: $27,11 \%$ ( $\mathrm{n}=$ 8689) "Doenças e Distúrbios do Sistema Respiratório" e 22,73\% ( $=7283)$ "Recém-nascidos e Neonatos com Afecções do Período Perinatal”. Por outro lado, destacam-se entre os casos cirúrgicos (n 
= I8684), os seguintes MDCs: 47,69\% (n = 8910) “Gravidez, Parto e Puerpério” e 23,99\% (n= 4482) "Doenças e Distúrbios do Sistema Musculoesquelético e Tecido Conjuntivo".

Tabela 3: Análise dos MDC's dos Hospitais Fhemig - Case Mix e Ineficiência Operacional do custo fixo por Componentes Clínico e Cirúrgico, 0I/07/2020 a 3I/07/202I.

\begin{tabular}{|c|c|c|c|c|c|c|c|c|}
\hline Tipo de DRG & & Clín & aicos & & & Cirúr & rgicos & \\
\hline Descrição do MDC & Altas & $\begin{array}{l}\% \text { de } \\
\text { altas }\end{array}$ & $\begin{array}{l}\text { Case } \\
\text { Mix }\end{array}$ & Inef & Altas & $\begin{array}{l}\% \text { de } \\
\text { altas }\end{array}$ & $\begin{array}{l}\text { Case } \\
\text { Mix }\end{array}$ & Inef \\
\hline Gravidez, Parto e Puerpério & 1300 & $4,06 \%$ & 0,8178 & $99,52 \%$ & 8910 & $47,69 \%$ & 0,8236 & $150,56 \%$ \\
\hline Doenças e Distúrbios do Sistema Respiratório & 8689 & $27, \mathrm{II} \%$ & 2,0423 & I I $5,06 \%$ & 446 & $2,39 \%$ & 2,3373 & $\mathrm{I} 53,74 \%$ \\
\hline $\begin{array}{l}\text { Doenças e Distúrbios do Sistema } \\
\text { Musculoesquelético e Tecido Conjuntivo }\end{array}$ & 3566 & $\mathrm{II}, \mathrm{I} 3 \%$ & 0,8543 & $190,01 \%$ & 4482 & $23,99 \%$ & $\mathrm{I}, 79 \mathrm{I} 8$ & $306,66 \%$ \\
\hline $\begin{array}{l}\text { Recém-nascidos e Neonatos com Afecções do } \\
\text { Período Perinatal }\end{array}$ & 7283 & $22,73 \%$ & I,I649 & $\mathrm{I} 56,94 \%$ & & $0,00 \%$ & & \\
\hline Doenças e Distúrbios do Sistema Nervoso & $2 \mathrm{I} 05$ & $6,57 \%$ & $\mathrm{I}, \mathrm{I} 270$ & $170,65 \%$ & 500 & $2,68 \%$ & 3,0383 & $162,52 \%$ \\
\hline Doenças e Distúrbios do Sistema Digestivo & $\mathrm{I} 276$ & $3,98 \%$ & 0,9639 & $203,69 \%$ & $\mathrm{I} 234$ & $6,60 \%$ & 1,6670 & $187,47 \%$ \\
\hline Doenças e Distúrbios do Sistema Circulatório & 1025 & $3,20 \%$ & 0,9457 & $166,26 \%$ & 199 & $1,07 \%$ & 2,6403 & $\mathrm{I} 49,75 \%$ \\
\hline $\begin{array}{l}\text { Doenças e Distúrbios da Pele, Tecido Celular } \\
\text { Subcutâneo e Mama }\end{array}$ & 660 & $2,06 \%$ & 0,9383 & I80,2I\% & 524 & $2,80 \%$ & $\mathrm{I}, 4606$ & $323,57 \%$ \\
\hline $\begin{array}{l}\text { Traumatismos, Intoxicações e Efeitos Tóxicos } \\
\text { de Drogas }\end{array}$ & 800 & $2,50 \%$ & 0,8448 & $\mathrm{I} 65,06 \%$ & 359 & $\mathrm{I}, 92 \%$ & I,9489 & $229,95 \%$ \\
\hline $\begin{array}{l}\text { Doenças e Distúrbios do Ouvido, Nariz, Boca } \\
\text { e Garganta }\end{array}$ & 572 & $\mathrm{I}, 78 \%$ & $0,884 \mathrm{I}$ & $\mathrm{I} 79,40 \%$ & 474 & $2,54 \%$ & I,5637 & $306,11 \%$ \\
\hline $\begin{array}{l}\text { Doenças Infecciosas e Parasitárias, Sistémicas } \\
\text { ou de Localização Não Específica }\end{array}$ & 824 & $2,57 \%$ & $\mathrm{I}, 289 \mathrm{I}$ & $\mathrm{I} 49,56 \%$ & II 2 & $0,60 \%$ & 3,9754 & $104,67 \%$ \\
\hline $\begin{array}{l}\text { Doenças e Distúrbios do Rim e do Trato } \\
\text { Urinário }\end{array}$ & 828 & $2,58 \%$ & 0,8726 & I94,8I\% & 77 & $0,41 \%$ & 2,0504 & $292,96 \%$ \\
\hline $\begin{array}{l}\text { Doenças e Distúrbios do Sistema Hepatobiliar } \\
\text { e Pâncreas }\end{array}$ & 583 & $\mathrm{I}, 82 \%$ & I,0I82 & $170,65 \%$ & 203 & $\mathrm{I}, 09 \%$ & I,8892 & $24 \mathrm{I}, 77 \%$ \\
\hline $\begin{array}{l}\text { Doenças e Distúrbios do Sistema Reprodutivo } \\
\text { Feminino }\end{array}$ & 263 & $0,82 \%$ & 0,7920 & I66,0I\% & $4 \mathrm{I} 4$ & $2,22 \%$ & I,I636 & $282,93 \%$ \\
\hline $\begin{array}{l}\text { Fatores com Influência no Estado de Saúde e } \\
\text { Outros Contatos com os Serviços de Saúde }\end{array}$ & 352 & $\mathrm{I}, \mathrm{I} 0 \%$ & 0,8655 & $483,74 \%$ & 294 & $\mathrm{I}, 57 \%$ & 2,1530 & $537,43 \%$ \\
\hline $\begin{array}{l}\text { Doenças e Distúrbios Endócrinos Nutricionais } \\
\text { e Metabólicos }\end{array}$ & 419 & $\mathrm{I}, 3 \mathrm{I} \%$ & 0,8558 & $243,38 \%$ & 70 & $0,37 \%$ & $\mathrm{I}, 4920$ & $224,92 \%$ \\
\hline $\begin{array}{l}\text { Doenças e Distúrbios do Sangue/Órgãos } \\
\text { Hematopoiéticos e Doenças Imunológicas }\end{array}$ & 409 & $\mathrm{I}, 28 \%$ & I,04I6 & $179,43 \%$ & 46 & $0,25 \%$ & 2,6578 & $\mathrm{I} 35,49 \%$ \\
\hline $\begin{array}{l}\text { Infecções pelo Vírus da Imunodeficiência } \\
\text { Humana }\end{array}$ & 416 & $\mathrm{I}, 30 \%$ & 2,0323 & $\mathrm{I} 64,8 \mathrm{I} \%$ & & $0,00 \%$ & & \\
\hline Queimaduras & 240 & $0,75 \%$ & $\mathrm{I}, 688 \mathrm{I}$ & $308,72 \%$ & 94 & $0,50 \%$ & 3,9247 & I09,II\% \\
\hline $\begin{array}{l}\text { Doenças e Distúrbios do Sistema Reprodutivo } \\
\text { Masculino }\end{array}$ & 58 & $0,18 \%$ & 0,9013 & $213,49 \%$ & 78 & $0,42 \%$ & $\mathrm{I}, 2865$ & $365,90 \%$ \\
\hline $\begin{array}{l}\text { Doenças e Distúrbios Mieloproliferativas e } \\
\text { Neoplasias Mal Diferenciadas }\end{array}$ & 87 & $0,27 \%$ & I,5398 & $\mathrm{I} 48,93 \%$ & 33 & $0,18 \%$ & $\mathrm{I}, 8360$ & $266,50 \%$ \\
\hline Doenças e Distúrbios do Olho & 37 & $0,12 \%$ & 0,7677 & $263,99 \%$ & 80 & $0,43 \%$ & I,4I29 & $290,69 \%$ \\
\hline $\begin{array}{l}\text { Uso de Álcool/Droga e Distúrbios Mentais } \\
\text { Orgânicos Induzidos por Álcool ou Droga }\end{array}$ & II6 & $0,36 \%$ & 0,9264 & I I $2,70 \%$ & & $0,00 \%$ & & \\
\hline Traumatismos múltiplos significativos & 64 & $0,20 \%$ & 2,0940 & I I $9,54 \%$ & 46 & $0,25 \%$ & 5,5507 & $\mathrm{I} 25,86 \%$ \\
\hline Doenças e Distúrbios Mentais & 76 & $0,24 \%$ & 1,0306 & $\mathrm{I} 85,30 \%$ & 9 & $0,05 \%$ & $3,5 \mathrm{IO0}$ & $360,72 \%$ \\
\hline Total geral & 32048 & $100,00 \%$ & 1,3159 & $\mathrm{I} 44,30 \%$ & 18684 & $100,00 \%$ & $\mathrm{I}, 3903$ & $199,36 \%$ \\
\hline
\end{tabular}

Fonte: Sistema DRG Brasil, compilado pelos autores (outubro/202I). Legenda: Inef: Ineficiência. 
Ao se tomar para análise as MDCs "Gravidez, Parto e Puerpério" juntamente com "Recémnascidos e Neonatos com Afecções do Período Perinatal”, identifica-se todos os DRGs que compõem a assistência Materno Infantil. Sob essa ótica, é possível analisar um perfil assistencial, também sob seu modelo de agrupamento em componente clínico e componente cirúrgico.

Ainda considerando a Tabela 3, evidencia-se para todas as MDCs, em que essa comparação é possível, é majoritariamente no componente cirúrgico que reside a maior ineficiência, o que permite alocação direcionada de esforços e possibilita resultados de melhorias mais rápidos, pois esse componente é dotado de maior padronização da assistência comparado ao componente clínico.

Esse modelo de análise permite avaliar, no conjunto dos serviços de um Hospital ou de uma Holding (Operadoras de Seguro Saúde, Cooperativas, Autogestão, Fhemig, etc.), esses componentes por seus diferenciais de performance (positivos ou negativos); orientar as políticas administrativas de alocação de recursos, direcionados pelas prioridades de gestão da alta administração, como anteviu o grupo de Yale sobre potenciais usos da metodologia DRG (FETTER, I980).

\section{Considerações finais sobre o processo de implantação da solução na Fhemig}

Por fim, é relevante abordar, sinteticamente, dois aspectos considerados críticos à introdução de qualquer Sistema de Informação em uma instituição:

- A contratação do Sistema assegura que a Fhemig é proprietária da totalidade dos dados (não tratados e tratados), assegurando a liberdade de escolha de fornecedores e das tecnologias de gestão desenvolvidas a partir da utilização dessas informações;

- Estima-se que os custos totais incorridos na implantação da Solução e da Metodologia DRG na Fhemig, incluindo a contratação pelo modelo de contrapartida, insumos de TI e custos de pessoal direcionados ao projeto, são muito superados pelos retornos econômicos e sociais. Se considerarmos o custo médio do leito hospitalar de R \$ 200,00, são projetados potenciais de economia anual superiores a R \$ I00 milhões de reais quando alcançadas as metas de desempenho e redução de condições adquiridas no conjunto dos IO hospitais Fhemig. E o custo social é a oportunidade de aumentar o acesso da população aos hospitais Fhemig, ao assegurar um potencial de oferta anual de 28400 admissões hospitalares (Excesso de diárias consumidas na assistência, relacionada à diferença de desempenho real e a meta, dividido pela Média de Permanencia prevista par o conjunto de DRGs assistidos no período).

\section{CONCLUSÃO}

A implantação do DRG na Fhemig e os resultados iniciais já alcançados refletem uma mudança de paradigma no planejamento, monitoramento e avaliação dos serviços hospitalares. $O$ uso da metodologia DRG na Fhemig trouxe como inovação a utilização do benchmark externo como direcionador das metas de ferramentas gerenciais tanto no campo das políticas e diretrizes da fundação, bem como no gerenciamento dos hospitais. Assegurar o alcance das metas fixadas a partir do DRG significará ampliação de acesso à atenção hospitalar para a população com perspectiva de melhoria da qualidade.

O diagnóstico do perfil assistencial apresentou os indicadores de complexidade e desempenho hospitalar da Fhemig, além de compará-los com os resultados do SUS, Saúde Suplementar e Assistência Privada, evidenciando o vocacionamento para especialidades com tendência para case mix maiores na Fhemig. Ademais, o processo de clusterização sequencial da metodologia DRG por MDC permite um olhar sobre conjuntos de serviços hospitalares.

A iniciativa apresentada do Projeto DRG pode ser replicada e adaptada. Primeiramente, deve-se avaliar o escopo de atuação. A implantação e uso do DRG permite avaliar o conjunto dos serviços de um Hospital, ou até mesmo de uma Holding, como a Fhemig, Operadoras de Seguro Saúde, Cooperativas, 
Autogestão, entre outros. No âmbito hospitalar, a principal estratégia de aprimoramento e multiplicação do uso do DRG é de utilização da ferramenta como insumo para a melhoria de processos assistenciais. Em relação à Fhemig, a perspectiva é de utilizar o DRG não só como ferramenta diagnóstica de perfil assistencial e de desempenho hospitalar, mas também utilizar todo o potencial da ferramenta de apoio gerencial para priorizar diretrizes assistenciais, monitoramento, intervenções e avaliação do desempenho assistencial.

Espera-se que este estudo de caso contribua com o aprimoramento em metodologias de avaliação da assistência hospitalar, em especial no Sistema Único de Saúde. Contudo, novos estudos precisam ser realizados nessa área para ampliar o uso da metodologia DRG no sistema de saúde brasileiro.

As limitações do estudo estão relacionadas ao objeto metodológico, estudo de caso, devido à dificuldade de generalização dos resultados obtidos, ou seja, as particularidades e especificidades na natureza do objeto de estudo podem não ser aplicáveis a outros fenômenos.

\section{REFERÊNCIAS BIBLIOGRÁFICAS}

BRASIL, Portaria GM/MS n ${ }^{\circ} 4.279$, de 30 de dezembro de 20I0. Estabelece diretrizes para a organização da Rede de Atenção à Saúde no âmbito do Sistema Único de Saúde. Brasília: Diário Oficial da União, 2010.

BRASIL, Portaria GM/MS N 3.390, de 30 de dezembro 2013. Dispõe sobre a Política Nacional de Atenção Hospitalar (PNHOSP) no âmbito do Sistema Único de Saúde (SUS), estabelecendo- se as diretrizes para a organização do componente hospitalar da Rede de Atenção à Saúde (RAS). Brasília: Diário Oficial da União, 2013.

BRASIL, Portaria de Consolidação $n^{\circ}$ 2, de 28 de setembro de 2017. Dispõe sobre Consolidação das normas sobre as políticas nacionais de saúde do Sistema Único de Saúde. Brasília: Diário Oficial da União, 2017.

BRASIL, Portaria de Consolidação $n^{\circ} 3$, de 28 de setembro de 2017. Dispõe sobre Consolidação das normas sobre as redes do Sistema Único de Saúde. Brasília: Diário Oficial da União, 2017.

AGÊNCIA NACIONAL DE SAÚDE SUPLEMENTAR. Guia para Implementação de Modelos de Remuneração baseados em valor. Rio de Janeiro: 2019.

CARPANEZ LR, MALIK AM. O efeito da municipalização no sistema hospitalar brasileiro: os hospitais de pequeno porte. Ciência \& Saúde Coletiva, 26(4):I289-I298, 202I. DOI: I0.I590/I4I38I23202I264.072420I9.

CARPANEZ, L.R, MALIK, A.M. O efeito da municipalização no sistema hospitalar brasileiro: Os hospitais de pequeno porte. Cien Saude Colet [periódico na internet] (2019/Jul). Disponível em: http://www.cienciaesaudecoletiva.com.br/artigos/o-efeito-da-municipalizacao-no-sistema-hospitalarbrasileiro-os-hospitais-de-pequeno-porte/I7293.

FETTER, R.B. Diagnosis Related Groups: Understanding Hospital Performance. Interfaces, I99 I, 2 I (I), 6-6I. Doi I0.1287.

FETTER, R.B.; SHIN, Y.; FREEMAN, J.L.; AVERILL, R. F.; THOMPSON J.D. Case Mix Definition by Diagnosis-Related Groups, Medical Care, Vol. I8, No. 2, Supplement: Case Mix Definition by Diagnosis-Related Groups (Feb., I980), pp. i+iii $+_{v}+$ ix + I-53 (57 pages). 
GIL, A.C. Métodos e Técnicas de Pesquisa Social. 6. ed. São Paulo: Atlas, 2008. 220 p.

LA FORGIA, G. M., COUTTOLENC, B.F. Desempenho Hospitalar no Brasil: A Busca pela Excelência. Washington, DC: Banco Mundial. 2008. 360p.

MENDES EV. As redes de atenção à saúde. Ciência \& Saúde Coletiva, I5(5):2297-2305, 2010. https://doi.org/I0.I590/SI4I3-8I232010000500005

NORONHA, M.F. et al. O desenvolvimento dos "Diagnosis Related Groups" - DRGs. Metodologia de classificação de pacientes hospitalares. Rev. Saúde pub., S. Paulo, 25: 198-208, I99I. doi.org/I0.1590/S0034-89101991000300007

PAIM JS \& TEIXEIRA CF. Política, planejamento e gestão em saúde: balanço do estado da arte. Rev. Saúde Pública. 2006;40(N Esp.):73-8. https://doi.org/I0.1590/S0034-89I020060004000I I

SANTOS, T. B. S.; PINTO, I. C. M. Política Nacional de Atenção Hospitalar: con(di)vergências entre normas, Conferências e estratégias do Executivo Federal. Saúde Debate. Rio De Janeiro, V. 4I, N. Especial 3, P. 99-II3, set 2017.

UGÁ MAD. Sistemas de alocação de recursos a prestadores de serviços de saúde - a experiência internacional. Ciência \& Saúde Coletiva, I7(I2):3437-3445, 2012.

YIN, R. K. Estudo de caso: planejamento e métodos. Porto Alegre: Bookman, 2010. 